\title{
An integrated fluorescence activated cell sorter fabricated by femtosecond laser micromachining
}

\author{
P. Paiè, F. Bragheri, R. Martinez Vazquez, N. Bellini, R. Ramponi, R. Osellame \\ Istituto di Fotonica e Nanotecnologie (IFN) - CNR and Dipartimento di Fisica - Politecnico di Milano, Milano, Italy \\ P. Minzioni, I.Cristiani \\ CNISM and Department of Electronics, University of Pavia, Via Ferrata 1,I-27100 Pavia, Italy \\ C. Mondello \\ Istituto di Genetica Molecolare (IGM) - CNR, Via Abbiategrasso 207, 27100 Pavia, Italy \\ petra.paie@mail.polimi.it
}

\begin{abstract}
We present here a fully integrated fluorescence activated cell sorter (FACS), able to perform analysis at single cell level. This optofluidic device is obtained on a fused silica substrate with the use of femtosecond laser micromachining.
\end{abstract}

\section{Introduction}

During the last few years we have assisted to innovative improvements in the fields of chemistry and biology thanks to the introduction and the development of Lab On Chip (LOC); LOCs are devices that use networks of microchannels to transport, mix, separate and analyze very small amount of sample.

An aspect that is gaining increasing interest is the capability to integrate in LOC systems the optical components needed for detection and analysis of biological samples [1]; this aspect is fundamental in order to achieve a compact and portable device with an extremely high accuracy in alignment between fluidic and optical components.

Moreover these optofluidic devices are powerful tools to analyze and manipulate sample at single cell level to unravel the hidden complexity in nominally homogeneous populations and this could be useful for early detection of several diseases. In fact populations of cells considered nominally identical, could present some heterogeneity that the traditional analyses, based on averaged data, are not able to investigate.

Among the different microfabrication technologies, femtosecond laser micromachining (FLM) is ideally suited for the manufacturing of fully integrated optofluidic devices [2]; in fact if combined with chemical etching it provides the integration of both microfluidic and optical functions on the same glass chip leading to monolithic, perfectly aligned, robust and portable devices. Moreover this technique has the advantage of guaranteeing a rapid prototyping of the device, and also thanks to the 3D nature of FLM, it is possible to fabricate innovative and unique device geometries.

\section{Discussion}

In this work we present the fabrication and the validation of a fully integrated optofluidic device, capable of sorting single cells with optical forces, on the basis of their fluorescence. The schematic of this device is shown in Fig.1.

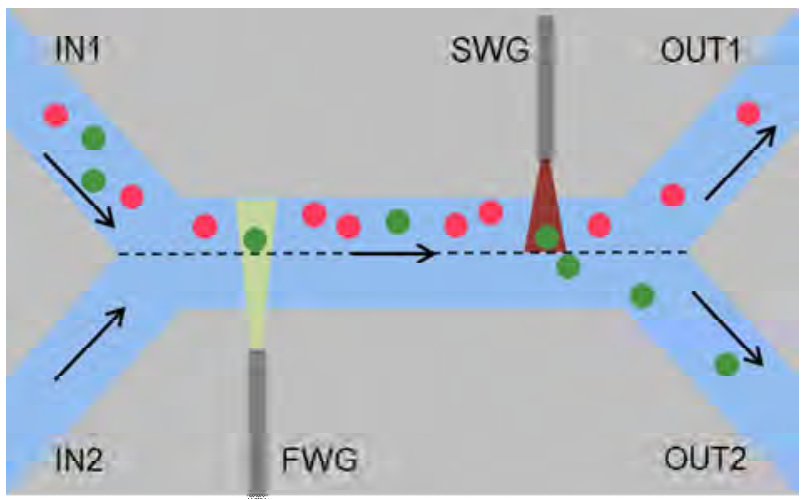

Fig. 1 Schematic diagram of the cell sorting working principle. Here the green particles represent the fluorescence ones, while FWG is the waveguide used to excite the fluorescence of the particles and the SWG is the waveguide used to separate the sample.

The design is based on a "X-shaped" microchannel, characterized by two input and two output channels, IN1 is used to insert the heterogeneous sample, while only buffer solution enters from IN2. These two fluids merge in the 
main channel without mixing thanks to the laminarity of the flux. In the central section of the device we perform both the fluorescence detection and the separation of the fluorescently labeled cells with the use of optical waveguides that orthogonally intersect the microchannel. With the first waveguide, coupled to a low-power visiblewavelength cw laser source, we are able to excite the fluorescence of the particles in the channel; depending on the fluorescence signal, the second waveguide, coupled to a high-power $\mathrm{cw}$ ytterbium fiber laser, is able to deflect the selected particle toward OUT2, using optical forces, while all the other particles keep flowing toward OUT1. This process allows one to efficiently separate the population with a single cell resolution.

As previously discussed, this device is fabricated by the use of femtosecond laser micromachining followed by chemical etching, in a substrate of fused silica.

Femtosecond laser irradiation is performed by a commercial femtosecond laser source (femtoREGEN, HIGHQlaser) that can provide pulses at a repetition rate up to $960 \mathrm{kHz}$, with an energy up to $23 \mu \mathrm{J}$ and a wavelength of $1040 \mathrm{~nm}$. For the fabrication of this device we have set the repetition rate at $500 \mathrm{kHz}$, the laser beam is frequency doubled and it is focused in the sample by a 50x, 0.6 N.A. microscope objective. Concerning the pulse energy and the scan speed we have to discriminate between the fabrication of microchannels and waveguides. In fact, the optimized parameters for microchannels are: a pulse energy of $700 \mathrm{~nJ}$ and a scan speed of $1 \mathrm{~mm} / \mathrm{s}$; for the waveguides, instead, the pulse energy used is $100 \mathrm{~nJ}$ and the scan speed is $0.1 \mathrm{~mm} / \mathrm{s}$.

The second step of the fabrication process is the chemical etching; here the device is exposed to a $20 \%$ aqueous solution of $\mathrm{HF}$ at $35^{\circ} \mathrm{C}$ in a sonic bath in order to obtain the formation of the microchannel.

The capability of the device to act as a micro fluorescence activated cell sorter has been tested first with polystyrene beads, and secondly with a cell sample of human transformed fibroblasts transfected with a plasmid encoding the enhanced green fluorescent protein (EGFP). Fig.2 shows a sequence of frames demonstrating the behaviour of the device when a non-fluorescent (top) and a fluorescent cell (bottom) are flowing in the channel.
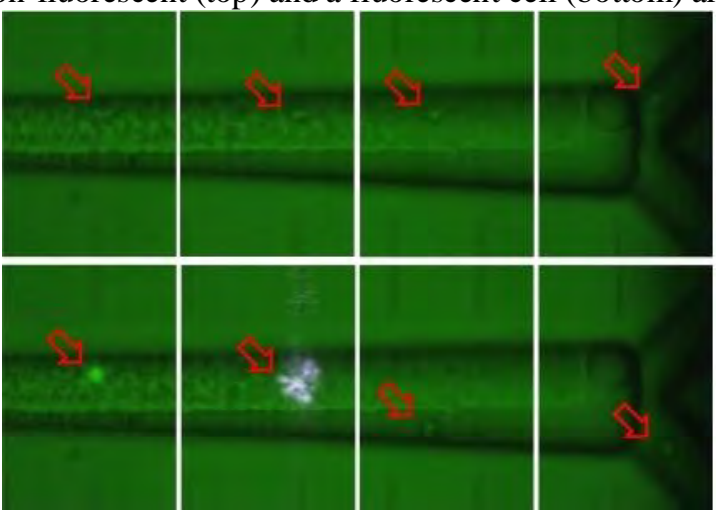

Fig. 2 Sequence of frames showing the capability of the device to separate, on the basis of their emitted fluorescence, a heterogeneous population of cells using radiation pressure.

As we can observe, the non-fluorescent cell proceeds in the upper side of the channel without being deflected by the SWG, exiting at OUT1, while the fluorescent cell is identified by the emission of the fluorescence signal at the first waveguide and is therefore pushed toward OUT2 by radiation pressure at the second waveguide, allowing the physical separation of the sample. We have automated this process with an appropriate software capable of revealing the emission of fluorescence and consequently switching on the ytterbium laser at the right moment.

\section{Conclusion}

Thanks to the capabilities of femtosecond laser micromachining we have fabricated an extremely portable and compact fluorescence activated cell sorter, completely based on optical forces. This device is able to perform an analysis at the single cell level in order to separate heterogeneous populations of cells, on the basis of their fluorescence signal, with high resolution and reliability.

\section{References}

[1] B. Kuswandi, J. Huskens, and W. Verboom. Optical sensing systems for microuidic devices: A review. Analytica chimica acta, 601:141\{155, 2007.

[2] R. Osellame et al., Laser Photonics Rev. 5, 442, 2011 